Check for updates

Cite this: RSC Adv., 2017, 7, 43483

\title{
A three dimensional sulfur/reduced graphene oxide with embedded carbon nanotubes composite as a binder-free, free-standing cathode for lithium-sulfur batteries $\dagger$
}

\begin{abstract}
Kongqing Ying, ${ }^{a}$ Ran Tian, ${ }^{a}$ Jie Zhou, ${ }^{a}$ Hua Li, (D) *ab Roberto Dugnani, ${ }^{c}$ Yanyan Lu, ${ }^{d}$ Huanan Duan, iD a Yiping Guo iD a and Hezhou Liu ${ }^{\text {ab }}$

Binder-free and free-standing electrodes have been regarded as an attractive and promising structure in lithium-sulfur batteries. In this work we describe how a free-standing sulfur/reduced graphene oxide with embedded carbon nanotubes electrode has been synthesized by a novel, facile and eco-friendly method taking advantage of the solubility difference of polar and nonpolar materials. First, the nonpolar elemental sulfur is dissolved in a weak polar solvent (ethanol) by intensive ultrasonication. During a subsequent heavy polar solvent (deionized water) drop-wise procedure, nano-sized sulfur particles are precipitated from the ethanol and deposited on the dispersed carbon nanotubes and graphene oxide. Noticeably, since ascorbic acid is taken as the reducing agent for graphene oxide at $75^{\circ} \mathrm{C}$, the process produces no toxic byproducts. Besides, the 'as-designed' sulfur/reduced graphene oxide with embedded carbon nanotubes graphene oxide displays a unique structure with both the sulfur and carbon nanotubes embedded in the basal plane of reduced graphene oxide. The manufactured electrode is found to exhibit excellent rate capability and cyclability, with the maximum capacity of $1025 \mathrm{~mA} \mathrm{~h} \mathrm{~g}^{-1}$ observed in the third cycle and a stable capacity of $704 \mathrm{~mA} \mathrm{~h} \mathrm{~g}^{-1}$ after 100 cycles.
\end{abstract}

Received 5th July 2017

Accepted 1st September 2017

DOI: $10.1039 / c 7 r a 07418 b$

rsc.li/rsc-advances

\section{Introduction}

With the development of portable devices, energy storage devices like rechargeable batteries are in high demand. Currently, in the portable devices' battery systems, lithium-ion batteries (LIBs) play a dominant role due to their high performance and being relatively light weight. But the energy density limits of LIBs cannot fit the increased demand of electric and large-scale vehicles. Researchers are exploring new energy storage devices such as lithium-sulfur batteries (Li-S batteries) ${ }^{1}$ and hydrogen storage, ${ }^{2}$ which could replace the commercial lithium-ion batteries due to their abundance and high energy density.

For Li-S batteries cathode, many kinds of carbon could act effectively as electric conductors to enhance the utilization of the insulating sulfur and could also serve as a porous framework to contain redox products. ${ }^{3-5}$ Various researchers have used

${ }^{a}$ State Key Laboratory of Metal Matrix Composites, School of Materials Science and Engineering, Shanghai Jiao Tong University, Dongchuan Road No. 800, Shanghai 200240, China. E-mail: lih@sjtu.edu.cn

${ }^{b}$ Collaborative Innovation Center for Advanced Ship and Deep-Sea Exploration, Shanghai Jiao Tong University, China

'University of Michigan-Shanghai Jiao Tong University Joint Institute, China

${ }^{d}$ Shanghai Petrochemical Academy, Shanghai 201512, China

$\dagger$ Electronic supplementary information (ESI) available. See DOI: $10.1039 / \mathrm{c} 7 \mathrm{ra} 07418 \mathrm{~b}$ different kinds of carbon to improve the property of S/C cathode as a result of the low density, abundance in nature, low cost, among other advantages of $\mathrm{Li}-\mathrm{S}$ batteries. $^{6-9}$ The volume changes during charging and discharging of $\mathrm{S} / \mathrm{C}$ cathode could be effectively suppressed because of the combination of the mesoporous carbon..$^{10}$ At the same period, carbon nanotubes (CNTs) have been introduced into Li-S batteries system with benefits such as its extremely high conductivity and low density. It has been shown that carbon nanotubes with $155{ }^{\circ} \mathrm{C}$ heat treatment did improve cell performance, by both an increase in discharge capacity and an enhancement in cycle life. This also indicated a new stabilization mechanism of sulfur in carbon. ${ }^{11-13}$ Unfortunately the loss of the sulfur cathode material causes significant capacity fading in lithium/sulfur cells which cannot be solved effectively by mesoporous carbon. Therefore, graphene oxide with reactive functional groups has been used to immobilize sulfur and lithium polysulfides via chemical approaches. In addition, the mechanism of polysulfide immobilization on defective graphene has also been studied., ${ }^{\mathbf{8} 14,15}$

Regretfully, the cathode materials of the composites mentioned above require the use of binders and aluminum foils as a part of the cell components. Conventionally designed electrodes use either PVDF (polyvinylidene fluoride) or PEO (poly (ethylene glycol)) binders. Moreover, the composites need to be ground into powder and then mixed with carbon black and 
polyvinylidene fluoride in $N$-methyl-2-pyrrolidone (NMP). ${ }^{16}$ Therefore, high energy density is difficult to achieve using this type of manufacturing.

Recently, researchers have given a lot of importance to a novel binder-free and current collector-free electrode designs. For instance, sulfur with activated carbon fibber cloth as a binder-free and free-standing cathode has been shown to be able to deliver a maximum discharge capacity of $1050 \mathrm{~mA} \mathrm{~h} \mathrm{~g}^{-1}$ while maintaining $800 \mathrm{~mA} \mathrm{~h} \mathrm{~g}{ }^{-1}$ after 80 cycles with only $33 \%$ sulfur content. ${ }^{17}$ Compared to carbon fiber, reduced graphene oxide with high specific surface areas is a more promising binder-free and free-standing carbon matrix. Wu et al. ${ }^{18}$ presented a 3D sulfur cathode embedded into porous reduced graphene oxide (rGO) with large areal mass loading of sulfur $\left(12 \mathrm{mg} \mathrm{cm}^{-2}\right)$ which displayed an extremely low decay rate (0.08\% per cycle after 300 cycles). Furthermore, Wu et al. ${ }^{\mathbf{1 9 , 2 0}}$ also presented a series of remarkable and interesting works of combination of rGO and $\mathrm{Li}_{2} \mathrm{~S}$ as binder-free and free-standing cathode which took advantage of both materials and exhibited an outstanding electrochemical performance. Besides, the scale of sulfur particles had a significant influence on the performance of $\mathrm{Li}-\mathrm{S}$ batteries. Ni et al. ${ }^{21}$ designed a facile route to fabricate an unique structure with multiscale and integrated S-particles in rGO, and the electrochemical performance was enhanced by the enhanced interfacial adhesion and conductivity plus shortened diffusion path. Wang et al. ${ }^{22}$ presented a flexible S-rGO sheet with $60 \%$ nano sulfur content. The specific discharge capacity is $800 \mathrm{~mA} \mathrm{~h} \mathrm{~g}^{-1}$ after 200 cycles, with current density $300 \mathrm{~mA} \mathrm{~h} \mathrm{~g}{ }^{-1}$, and the capacity fading rate only $0.035 \%$ per cycle. In Wang's work, nano sulfur is used directly increasing significantly the manufacturing cost of the electrodes, and the shape of the electrodes was restricted to circular disks on account of the manufacturing method used. Furthermore, enhancing the electrochemical performances by combination of CNTs and graphene is a very appealing method. Ding et $a .^{23}$ reported highly nitrogen doped CNT-graphene 3D nanostructures with a capacity retention of $97 \%$ after 200 cycles at a high rate of $2 \mathrm{C}$. $\mathrm{Hu}$ et al. ${ }^{24}$ presented a $3 \mathrm{D}$ GF-rGO hybrid nested hierarchical network which combined the advantages of graphene, rGO, porous materials and 3D network structure. This kind of cathodes with a sulfur loading of $9.8 \mathrm{mg} \mathrm{cm}^{-2}$ and sulfur content of $83 \mathrm{wt} \%$ exhibits a high areal capacity of $10.3 \mathrm{~mA} \mathrm{~h} \mathrm{~cm}^{-2}$ at $0.2 \mathrm{C}$ rate, which is extraordinary. In addition to the structures mentioned above, Yang et al. ${ }^{25}$ reported an interesting and flexible electrodes of stringed hollow nitrogendoped (N-doped) carbon nanospheres with a remarkably high energy density of $754 \mathrm{~W} \mathrm{~h} \mathrm{~kg}^{-1}$, which combined the advantages of hollow structures, 3D electrodes and flexible devices.

Besides, the $\mathrm{pH}$ of the solution is of great importance for the reducing of graphene oxide. The alkaline solution environment obtained when using the most common sulfur sources of $\mathrm{Na}_{2} \mathrm{~S}$, is not beneficial for the formation of graphene sponges due to the increased electrostatic repulsion between the rGO sheets. ${ }^{26}$ Therefore, most of methods of synthesis of rGO sponges are followed by sulfur loading, which make graphene sponges displaying a relatively loose structure with large pores formed by linked graphene sheets in the range of tens of micrometers.
Moreover, the intermediate production of the $\mathrm{Na}_{2} \mathrm{~S}_{2} \mathrm{O}_{3},{ }^{27} \mathrm{CS}_{2}$ (ref. 24) from most manufacturing methods produces poisonous gas.

In this work, a facile in situ method was used for preparing three-dimensional porous graphene/carbon nanotubes composite containing sulfur nanoparticles which act as binder-free and freestanding electrodes. With the proposed novel and environmentfriendly method, a 3D interconnected network porous structure with small pores of rGO combined with CNTs was formed. The manufactured structure not only could utilize graphene oxide to trap the soluble polysulfides by physical confinement and chemical bonding, but also could accommodate the volume changes during cycling. Additionally, it could also increase the number of conductive paths and enhance the conductivity because of CNTs. Besides, because of the use of ethanol and deionized water (DI water) only, this method was very suitable for industrial production due to its low cost and eco-friendly characteristics. Furthermore the proposed method allowed the design of various structural shapes with great ease. Finally, the designed sulfur/ reduced graphene oxide with embedded carbon nanotubes (S-CNTs@rGO) was shown to exhibit excellent rate capability and cyclability with sulfur and CNTs embedded in the basal plane of rGO.

\section{Experimental procedure}

\section{Synthesis of graphene oxide dispersion and preparation of CNTs}

Graphene oxide (GO) dispersion and acidization of CNTs were similar to what previously reported. ${ }^{28,29} \mathrm{CNTs}$ (purity > $95 \mathrm{wt} \%$ ) were purchased from Chengdu Organic Chemistry Co. Ltd., Chinese Academy of Sciences (Chengdu, China).

\section{Preparation of S/CNTs/ethanol solution}

$120 \mathrm{mg}$ elemental (nonpolar) sulfur and $18 \mathrm{mg}$ CNTs were dissolved for 2 hour in $120 \mathrm{~mL}$ ethanol with intensive ultrasonication at $35{ }^{\circ} \mathrm{C}$. Sulfur (purity $>99.5 \mathrm{wt} \%$ ) was purchased from Sinopharm Chemical Reagent Co., Ltd.

\section{Synthesis of S-CNTs@rGO}

$9 \mathrm{~mL}$ graphene oxide $\left(7 \mathrm{mg} \mathrm{mL}{ }^{-1}\right.$ ) was mixed with $12 \mathrm{~mL}$ DI water before adding drop-wise into $120 \mathrm{~mL}$ S/ethanol solution (mass ratio of graphene oxide and $S 1: 2$ ). Subsequently, the solution was heated to $65{ }^{\circ} \mathrm{C}$ to let the ethanol slowly evaporate until $20 \mathrm{~mL}$ solution was left and to ensure that the graphene oxide was at a proper concentration. In order to achieve homogeneous dispersion, stirring was carried out for more than 24 hours. Afterwards, $180 \mathrm{mg}$ of ascorbic acid powder was added into the mixture and stirred for an additional 30 minutes. The solution was poured into a mold and placed for 4 hours into a draught drying cabinet at $75{ }^{\circ} \mathrm{C}$ to obtain 3D graphene hydrogels. Subsequently, the monolith was taken out and put in de-ionized water for 24 hours, and finally freeze-dried for 48 hours into graphene aerogel.

\section{Material characterization}

The crystal structures of the as-prepared samples were characterized using powder X-ray diffraction (XRD) on a Goniometer 
Ultima IV (185 $\mathrm{mm})$ diffractometer with $\mathrm{Cu} \mathrm{K} \alpha$ radiation $(\lambda=$ $1.5418 \AA$ ) at a step of $5^{\circ}$ per minute. The morphology of samples was characterized by transmission electron microscope (JEM2100F, JEOL). Field-emission scanning electron microscope (FE-SEM) analysis and energy dispersive spectrometer (EDS) were performed on JSM-6700F at an acceleration voltage of 10.0 $\mathrm{kV}$. X-ray photoelectron spectroscopic (XPS) measurements were performed on a Kratos AXIS Ultra DLD spectrometer with a monochromatic AlK $\alpha$ X-ray source. Elemental Analysis (EA) was performed on VARIO EL cube (Elementar). Thermal gravimetric analysis (TGA) was conducted in nitrogen atmosphere at a heating rate of $10{ }^{\circ} \mathrm{C} \mathrm{min}^{-1}$ from $25{ }^{\circ} \mathrm{C}$ to $600{ }^{\circ} \mathrm{C}$.

\section{Electrochemical measurements}

The electrochemical properties of the electrodes were characterized at room temperature. A Li foil was used as the counter electrode. CR2025-type cell assembly was carried out in a Mikrouna glovebox with the moisture and oxygen concentration kept below $0.1 \mathrm{ppm}$. The Celgard 2400 microporous membrane was used as the separator. The electrolyte was approximately $1 \mathrm{M}$ LiTFSI/DOL + DME (1:1 by volume) with 1 wt\% $\mathrm{LiNO}_{3}$ additive.

The galvanostatic cycling test was done at a voltage range between $3.0 \mathrm{~V}$ and $1.6 \mathrm{~V}$ using a LAND CT2001A battery tester. Cyclic voltammetry (CV) was performed on an electrochemical workstation (Bio-Logic, France) in a voltage range of 1.6-3.0 V vs. $\mathrm{Li} / \mathrm{Li}^{+}$with a scan rate of $0.2 \mathrm{mV} \mathrm{s}^{-1}$. Electrochemical impedance spectroscopy measurement was performed over a frequency range from $100 \mathrm{kHz}$ to $0.01 \mathrm{~Hz}$ with an AC voltage of $5 \mathrm{mV}$ in amplitude using a Bio-Logic VMP-300 Battery Testing System.

\section{Results and discussion}

Fig. 1 showed the schematic diagram for the above described synthesis of 3D S-CNTs@rGO. The nonpolar elemental sulfur was dissolved in one weak polar solvent (i.e., ethanol) by

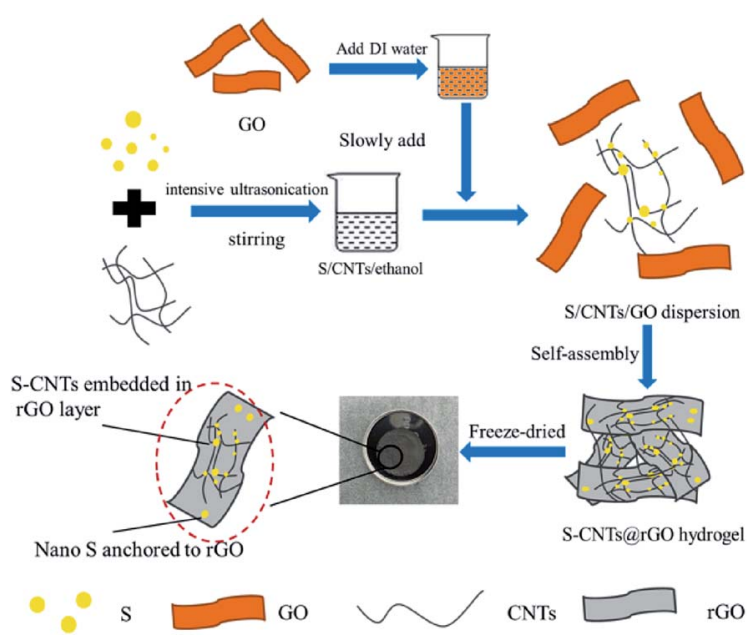

Fig. 1 Schematic of the 3D S-CNTs@arGO composite preparation. intensive ultrasonication. During heavy polar solvent (DI water) drop-wise procedure, nano-sized sulfur particles precipitated from the ethanol and deposited on the dispersive CNT and graphene oxide that provided abundant adhesion location for sulfur deposition. Subsequently, ascorbic acid, which was non-toxic and eco-friendly, was used as reducing agent to turn S-CNTs@GO into S-CNTs@rGO in a cylindrical mold.

The mechanism and influence factors for turning the sulfur particle with large size to nano size are discussed. According to the discussion of Fig. S1 and S2 in the ESI, $\uparrow$ the following conclusions can be obtained. The preferable ratio of water to S/ethanol solution was approximately $1: 5$ by volume. Besides, the supporting medium can provide abundant adhesion location for sulfur deposition, which is important for turning the sulfur particle with large size to nano size. Furthermore, the dropping rate is an important influence factor. By low dropping rate, nano $S$ particle rather than large $S$ particle will be slowly precipitated from the ethanol and anchor on the supporting medium. Therefore, low dropping rate will be beneficial for preparing nano size S particle. Besides, CNTs are able to relieve the $\mathrm{S}$ agglomeration problem. Therefore, it will be a good choice to take both CNTs and rGO as the supporting mediums to preparing the electrode of $\mathrm{Li}-\mathrm{S}$ batteries. And the discussion details are in the ESI. $\dagger$

Table 1 showed details of typical solution-based methods for sulfur preparation in $\mathrm{Li}-\mathrm{S}$ batteries compared with the one proposed in this work. It was found that most of the typical solution-based method described in the literature cannot avoid producing toxic gases. Additionally, most solution-based methods cannot produce nano-scaled sulfur in the composites. Comparatively the method proposed in this work is more eco-friendly than solution-based methods. As for fabricating binder-free structure, comparing to the solvothermal reaction and vacuum filtration procedure, our method is more flexible as it allows the fabrication of any monolithic shape (Fig. 2) rather than being limited to thin sheets. ${ }^{27,33,36,37}$

Fig. 2a-c show the free-standing 3D S-CNTs@rGO is wellshaped and suitable for common electrochemical measurements without any cutting or post-pressing procedure. Fig. 1d shows different shapes and designs can be easily obtained implementing the proposed method. Another advantage of the current method is that it could concurrently function as both energy storage and structural component. And the 3D S/rGO was prepared for comparison. And ESI Fig. S3a-d† online show that the cuboid shape obtained by the proposed method can have a good mechanical properties and elasticity.

The morphology and microstructure of the 3D S-CNTs@rGO were investigated by SEM and TEM (Fig. 3). The Fig. 3a and b display a tight structure with pore sizes in the range $3-12 \mu \mathrm{m}$. And the tight structure can also be found in 3D rGO according to the SEM image of 3D rGO (ESI Fig. S4 $\uparrow$ online). As mentioned above, the small size of pores result from the neutral solution environment. When more commonly used sulfur sources such as $\mathrm{Na}_{2} \mathrm{~S}$ is used, the alkaline solution environment does not promote the formation of graphene sponges due to the increased electrostatic repulsion between the rGO sheets. ${ }^{26}$ Moreover, the small size pores in the 3D network structure allow 
Table 1 Comparison of solution-based methods for S preparation in Li-S batteries

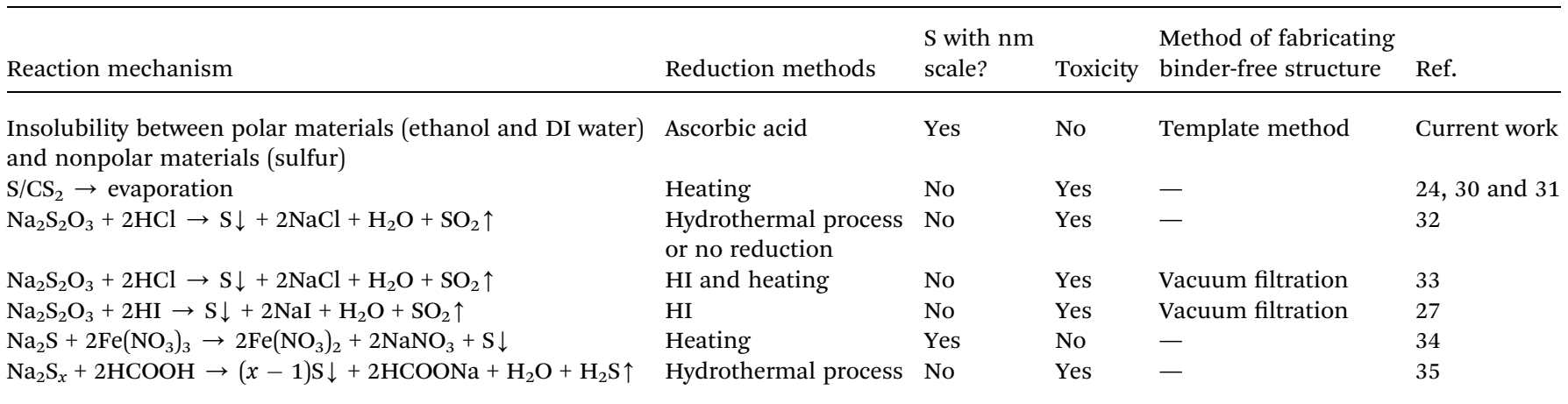

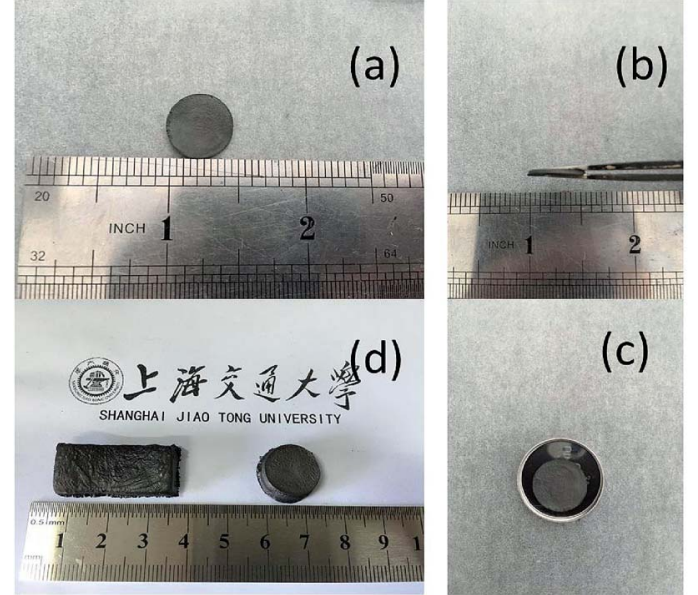

Fig. 2 (a-c) Photographs of free-standing 3D S-CNTsarGO sheet. (d) Different shapes and designs of the free-standing 3D S-CNTs@arGO.

efficient ion diffusion/mass transfer and could also be used as buffering/absorption reservoir for polysulphides. ${ }^{38}$ The SEM images also suggest the absence of widespread bulk sulfur, which indicates that with the help of the embedded CNTs, nano-sulfur either embedded or anchored uniformly onto the 3D CNTs@rGO network. Fig. 3c and d show the morphology of CNTs@rGO network. The CNTs embedded in the basal plane of rGO well and can act as conductive paths, for instance, in the case of the bridge connecting two rGO layers, hence enhancing the conductivity of the structure.

The morphology of sulfur nanoparticles and 3D S/rGO synthesized by this method were investigated by TEM (Fig. 3e and $\mathrm{f}$ ). As it can be seen from Fig. 3e S particles synthesized by this solution-based method are spherical with the diameters approximately from 20 to $200 \mathrm{~nm}$. As it can be appreciated in Fig. 3f, nano S particles are uniformly and strongly anchored on rGO sheets but the nanoparticles are easily agglomerated together. As the images of the 3D S-CNTs@rGO show in Fig. 3g and $\mathrm{h}$ with the assistance of CNTs, nano $\mathrm{S}$ particles can anchor mostly on the CNTs and then anchor or embed in the rGO layer without severe agglomeration. This implies that better dispersion, shorter conductive paths, stronger adsorption energy of the CNTs@rGO network and nano scaled restriction can be achieved. And the XRD patterns of the S/rGO and S-CNTs@rGO shown in ESI Fig. S5† online agree well with the patterns of standard sulfur (JCPSD no. 08-0247). Compared with the patterns of the S/rGO and S-CNTs@rGO, the XRD pattern of S-

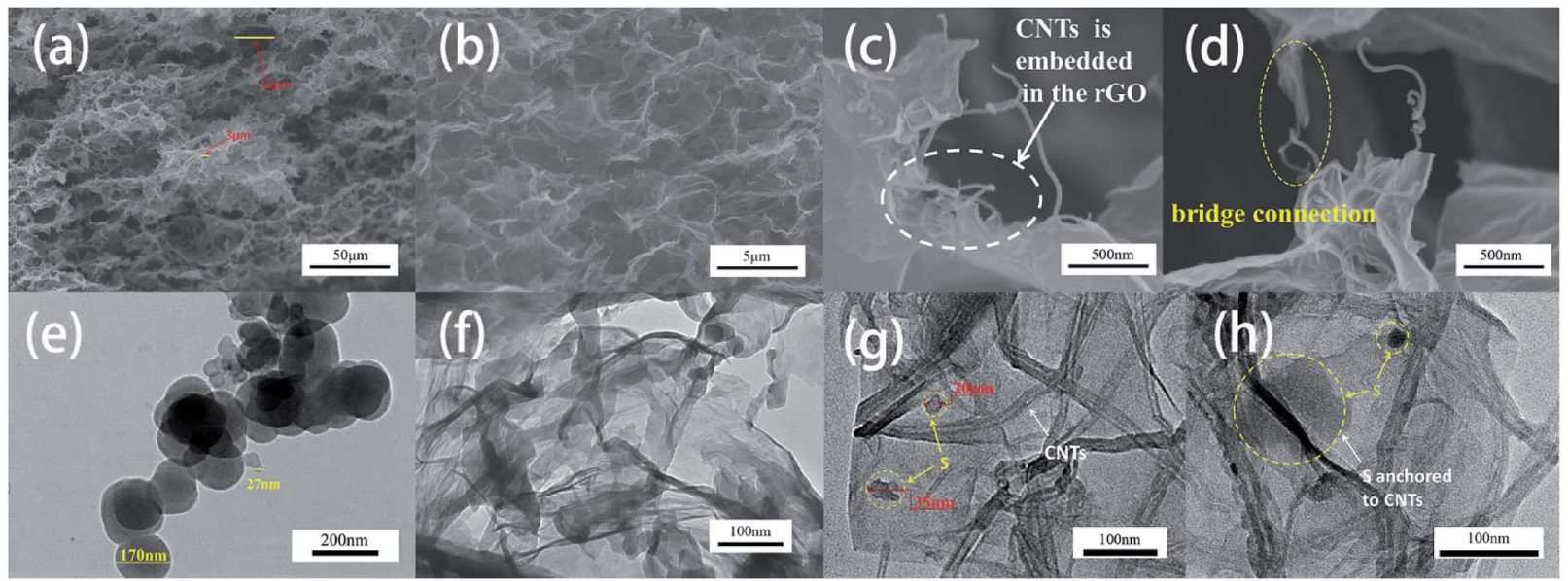

Fig. 3 (a-d) SEM images of morphology and microstructure of the 3D S-CNTs@rGO, (e) TEM image of sulfur nanoparticles precipitated from the ethanol, (f) TEM image of 3D S/rGO, (g) and (h) TEM images of the 3D S-CNTsarGO. 
CNTs@rGO exhibits peaks of sulfur with lower intensity, which can be attributed to its unique structure.

Fig. $3 \mathrm{a}$ and $\mathrm{b}$ show the morphology and microstructure of the 3D S-CNTs@rGO. Based on these images, no bulk sulfur is found; this phenomenon can be attributed to the fact that with the help of embedded CNTs, nano sulfur can also embed or anchor in the 3D CNTs@rGO network with good dispersion. In order to prove this point, elemental mapping of the 3D structure (Fig. 4a-d) were taken. As displayed in the SEM images (Fig. 4ad), although no bulk sulfur is found in Fig. 4a, the corresponding elemental mapping images show matched uniform spatial distribution of sulfur and carbon.

To investigate the electrochemical properties of the 3D SCNTs@rGO cathode, the sulfur content in the 3D S-CNTs@rGO and 3D S/rGO were measured by TGA measurement. According to the weight loss rate at $500{ }^{\circ} \mathrm{C}$ in Fig. 4 e, the sulfur content of 3D S-CNTs@rGO and 3D S/rGO are $55 \mathrm{wt} \%$ and $60 \mathrm{wt} \%$, respectively.

X-ray photoelectron spectroscopy (XPS) was applied to investigate the surface chemical composition of the 3D S-CNTs@rGO. According to the survey spectra of 3D SCNTs@rGO in Fig. 5a, only $\mathrm{C}, \mathrm{O}$ and $\mathrm{S}$ are found in the untreated sample. As displayed in Fig. $5 b$, the spectrum of $S 2 p$ can be fitted by two components. Each component has a $\mathrm{S} 2 \mathrm{p}_{3 / 2}$ and $2 \mathrm{p}_{1 / 2}$ doublet with an intensity ratio of $2: 1$. The binding energies of $\mathrm{S} 2 \mathrm{p}_{3 / 2}$ were $163.9 \mathrm{eV}$ and $164.3 \mathrm{eV}$, which can be ascribed to $\mathrm{C}-\mathrm{S} / \mathrm{S}-\mathrm{S}^{38,39}$ and $\mathrm{S}-\mathrm{O},{ }^{40}$ respectively. As for the peak at $168.6 \mathrm{eV}$, it can be ascribed to the sulfate species formed by the oxidation of sulfur in air. Furthermore, the intensity of the component peak at $168.6 \mathrm{eV}$ is relatively low compared to what found in the literature, ${ }^{22,40}$ which indicates that the synthesize method is mild and benefitted from the use of active materials. According to Fig. 5c, three component peaks at 284.8, 285.4, and $287.6 \mathrm{eV}$ are present in the $\mathrm{C} 1 \mathrm{~s}$ profile, which can be attributed to $\mathrm{sp}^{2} \mathrm{C}=\mathrm{C}, \mathrm{C}-\mathrm{S}$ and $\mathrm{C}=\mathrm{O} .{ }^{22,39}$ This observation, together with $\mathrm{S}$ $2 p$ result, supports the idea that chemical connections exists between the S and CNT@rGO. The XPS result of O 1s in Fig. 5d shows the component peaks at 531.3, 531.9 and $533.4 \mathrm{eV}$, which can be assigned to $\mathrm{C}=\mathrm{O}, \mathrm{S}-\mathrm{O}$ and $\mathrm{C}-\mathrm{OH} / \mathrm{C}-\mathrm{O}-\mathrm{C} .{ }^{22}$ The existence of strong $\mathrm{S}-\mathrm{O}$ indicates the intimately connection between $\mathrm{S}$ and graphene, which is also helpful for the immobilization of $S$ and polysulfides and the performance of the Li-S battery. ${ }^{41}$

Fig. 6a shows the cycling performance of the 3D SCNTs@rGO and S/rGO at $0.1 \mathrm{~A} \mathrm{~g}^{-1}$. The discharge capacity of the first cycle of 3D S-CNTs@rGO is $921 \mathrm{~mA} \mathrm{~h} \mathrm{~g}{ }^{-1}$. Then a capacity increase is observed in the following cycles. The maximum capacity of $1025 \mathrm{~mA} \mathrm{~h} \mathrm{~g}^{-1}$ was observed at the third cycle. The increase in capacity can be attributed to the gradual activation of the active materials. After 100 cycles, the observed capacity is $704 \mathrm{~mA} \mathrm{~h} \mathrm{~g}{ }^{-1}$, which is considerably more stable than the one of $\mathrm{S} / \mathrm{rGO}$. It can be seen in the Fig. 6 a that $\mathrm{S} / \mathrm{rGO}$ with high coulombic efficiency shows a poor cycling stability. During the $2^{\text {nd }}$ to $40^{\text {th }}$ cycles, although the coulombic efficiency of $\mathrm{S} / \mathrm{rGO}$ is $\sim 99 \%$, the charge capacity of every cycle is lower than the discharge capacity of its previous cycle and capacity loss are very obvious, which means the irreversible loss of soluble polysulfides intermediate is serious. As for the 3D S-
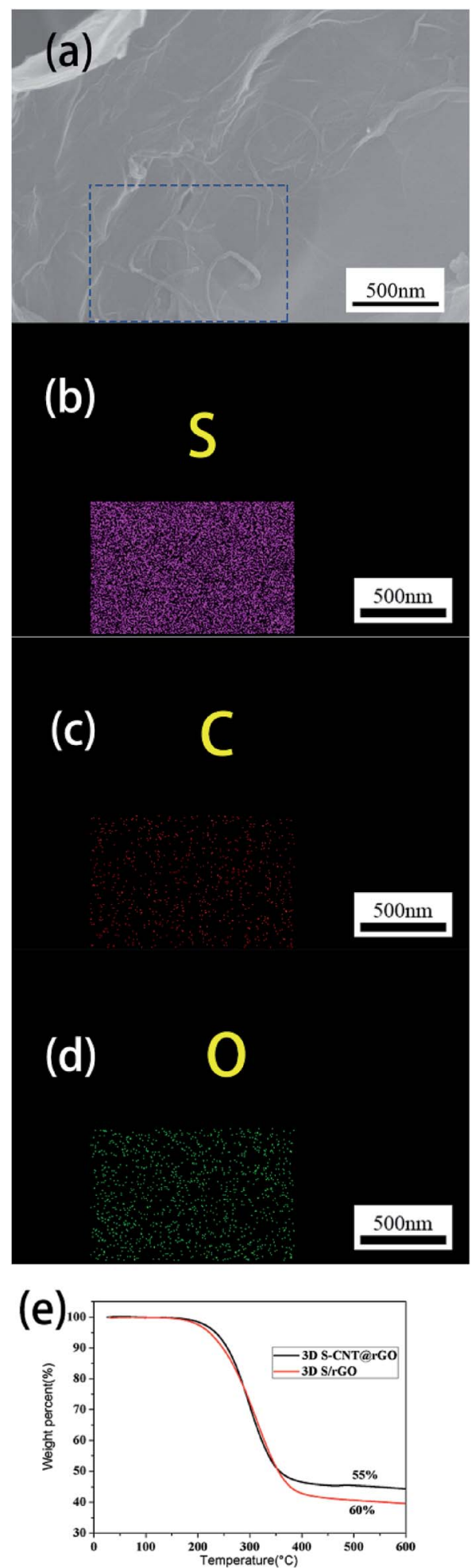

Fig. 4 (a-d) SEM image and the corresponding elemental mapping of 3D S-CNTs@rGO. (e) TGA curves for 3D S-CNTs@rGO and 3D S/rGO.

CNTs@rGO, its coulombic efficiency is $92 \%-97 \%$, which is relatively lower than $\mathrm{S} / \mathrm{rGO}$ but still is a good performance. And the relatively low coulombic efficiency results from the shuttle effects. During the $2^{\text {nd }}$ to $100^{\text {th }}$ cycles, its charge capacity of every cycle is higher than the discharge capacity of its previous 

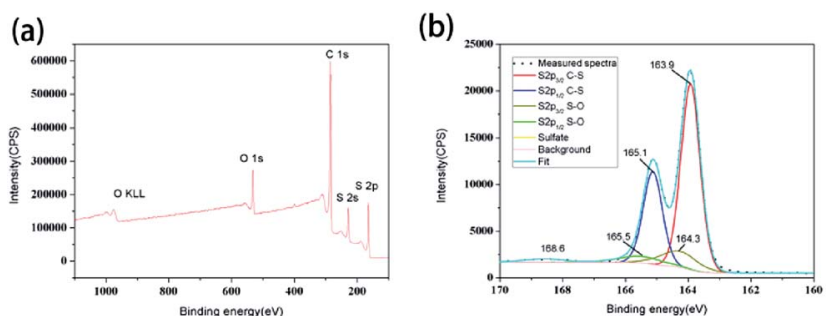

(c)
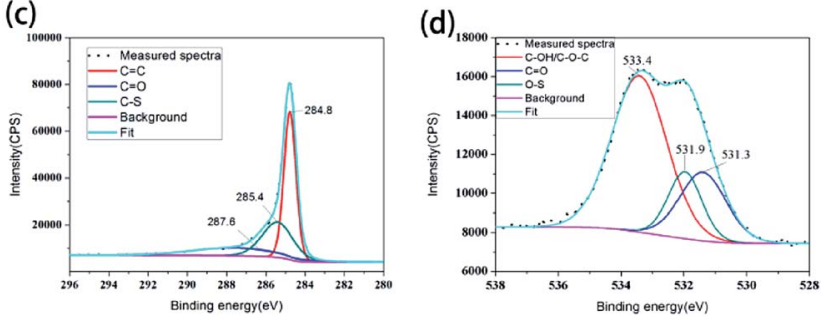

Fig. 5 (a) XPS survey spectra of the 3D S-CNTs@rGO. (b) S 2p, (c) C 1s, (d) O 1s spectra of the 3D S-CNTs@rGO

cycle, which is the evidence for the shuttle effects. Besides, the cycling stability of the S-CNTs@rGO is much better than the S/ rGO and the capacity loss is not very obvious during the charge/ discharge cycles, which indicates the good suppression of the irreversible loss of soluble polysulfides intermediate. The enhanced cycling stability can be attributed to the introduced CNTs which promote additional active locations for the deposition of nano sulfur. CNTs also result in a good dispersion and effective connection between nano sulfur and CNTs@rGO, which not only improve the electrical conductivity but also suppress polysulfides dissolution into the electrolyte..$^{42}$ Another reason for the observed superior performance of the electrode is its flexible framework. Since the graphene wafers prepared are porous and elastic they could accommodate the sulfur volume expansion during the charge/discharge cycles. From the corresponding charge/discharge profiles in Fig. 6b, typical profiles of lithium-sulfur batteries are observed. And the profiles are relatively stable, which also proves the excellent electrochemical stability of the 3D S-CNTs@rGO composite.

Cyclic voltammetry (CV) was used in the voltage range of 1.6$3.0 \mathrm{~V}$ at a sweep rate of $0.2 \mathrm{mV} \mathrm{s}^{-1}$ as shown in Fig. 6c. Two reduction peaks approximately at 2.3 and $2.0 \mathrm{~V}$ can be observed. These peaks can be attributed to the reduction of sulfur to soluble polysulfides intermediate, and to insoluble $\mathrm{Li}_{2} \mathrm{~S}_{2}$ and $\mathrm{Li}_{2} \mathrm{~S}$, suggesting the multiple reaction of sulfur with lithium ions. Meanwhile, the oxidation peaks at $2.45 \mathrm{~V}$ indicate the oxidation of insoluble $\mathrm{Li}_{2} \mathrm{~S}_{2}$ and $\mathrm{Li}_{2} \mathrm{~S}$ to polysulfides and to elemental sulfur. Furthermore, the overlapping of the 3-8 cyclic voltammetry curves certifies the excellent electrochemical stability of the 3D S-CNTs@rGO composite, which is in accordance with the observations made about Fig. $6 \mathrm{~b}$.

In this section we evaluate the rate performance of the S-CNTs@rGO composite by increasing the charge/discharge current density stepwise from $0.1 \mathrm{~A} \mathrm{~g}^{-1}, 0.2 \mathrm{~A} \mathrm{~g}^{-1}, 0.5 \mathrm{~A} \mathrm{~g}^{-1}$, $1.0 \mathrm{~A} \mathrm{~g}^{-1}, 1.5 \mathrm{~A} \mathrm{~g}^{-1}, 0.2 \mathrm{~A} \mathrm{~g}^{-1}$. As shown in Fig. $6 \mathrm{~d}$, the electrode delivers a discharge capacity of $1059 \mathrm{~mA} \mathrm{~h} \mathrm{~g}^{-1}$ at $0.1 \mathrm{~A} \mathrm{~g}^{-1}$, $834.5 \mathrm{~mA} \mathrm{~h} \mathrm{~g}^{-1}$ at $0.2 \mathrm{~A} \mathrm{~g}^{-1}, 589.7 \mathrm{~mA} \mathrm{~h} \mathrm{~g}^{-1}$ at $0.5 \mathrm{~A} \mathrm{~g}^{-1}$,

\section{(a)}

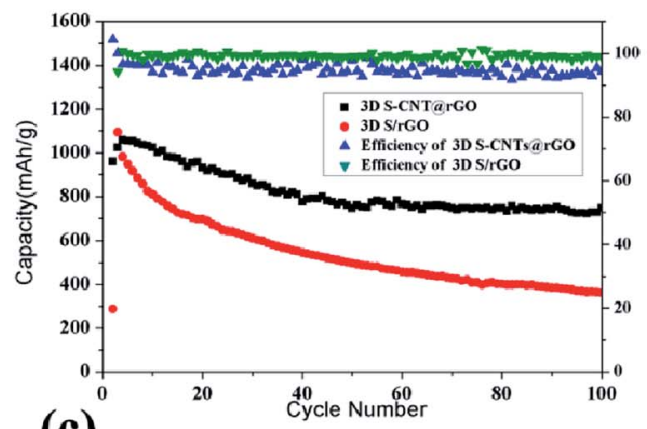

(c)

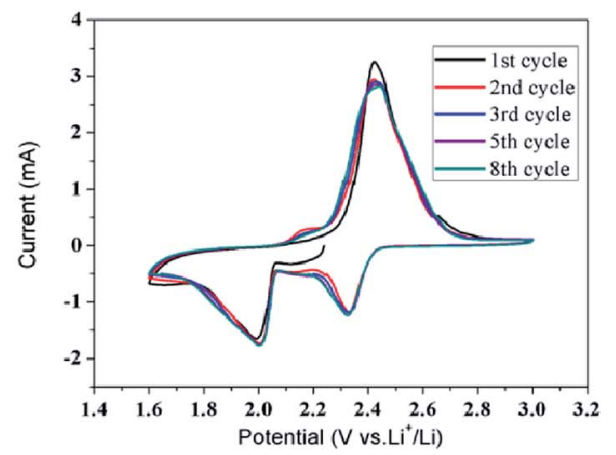

(b)

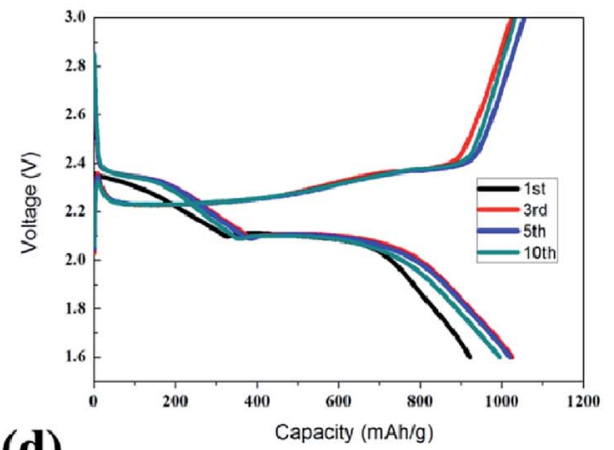

(d)

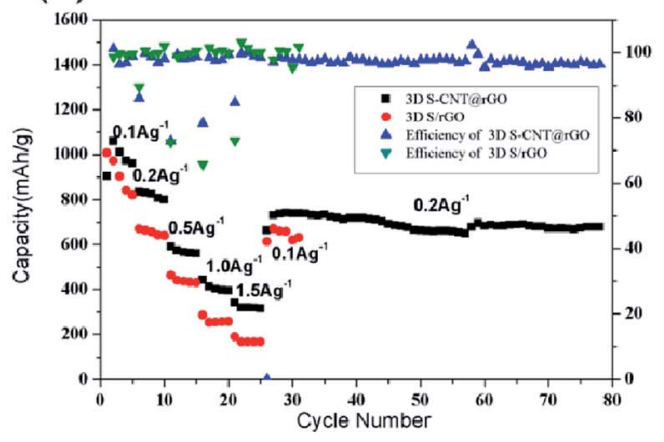

Fig. 6 Electrochemical performance of the 3D S-CNTs@rGO and 3D S/rGO: (a) cycling performance of the 3D S-CNTs@rGO and 3D S/rGO at $0.1 \mathrm{~A} \mathrm{~g}^{-1}$; (b) charge-discharge profiles of the 3D S-CNTs(arGO at $0.1 \mathrm{~A} \mathrm{~g}^{-1}$; (c) CV curve of the 3D S-CNTs(arGO at a sweep rate of 0.2 mV s (d) rate performances of 3D S-CNTsarGO and 3D S/rGO at stepwise current rates. 


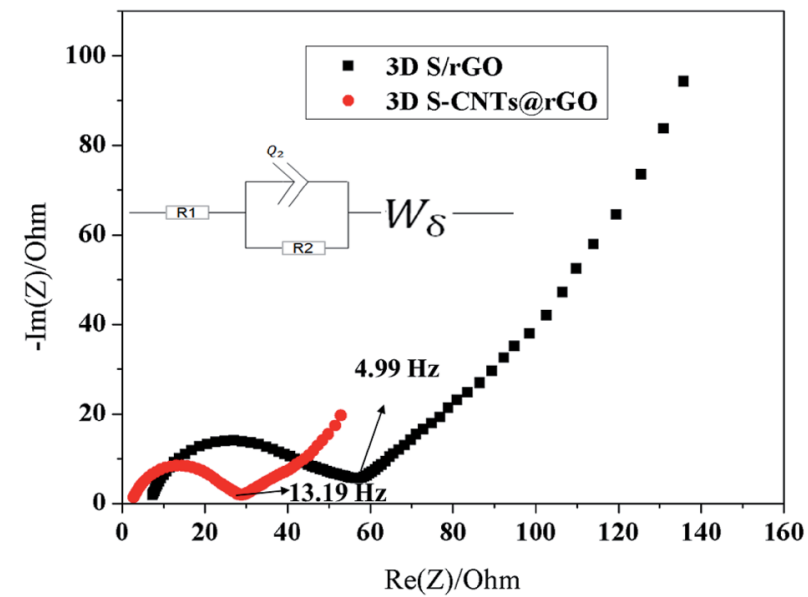

Fig. 7 EIS spectra of the 3D S-CNTs arGO and 3D S/rGO electrodes.

$441.8 \mathrm{~mA} \mathrm{~h} \mathrm{~g}^{-1}$ at $1 \mathrm{~A} \mathrm{~g}^{-1}, 339.8 \mathrm{~mA} \mathrm{~h} \mathrm{~g}^{-1}$ at $1.5 \mathrm{~A} \mathrm{~g}^{-1}$, $741.2 \mathrm{~mA} \mathrm{~h} \mathrm{~g}^{-1}$ at $0.2 \mathrm{~A} \mathrm{~g}^{-1}$. Comparing these results to the S/rGO, S-CNTs@rGO shows a better rate performance. These results indicate that the CNTs@rGO network can increase effective pathways for fast ion diffusion and electron transportation hence promoting the CNTs@rGO network's improved rate performance. Besides, it is noted that the electrode becomes very stable at $0.2 \mathrm{~A} \mathrm{~g}^{-1}$. And the cycling performance of the $3 \mathrm{D} \mathrm{S}-$ CNTs@rGO at $0.2 \mathrm{~A} \mathrm{~g}^{-1}$ in ESI Fig. S6 $†$ online also shows a very stable cycling performance. The discharge capacity of the first cycle is $839 \mathrm{~mA} \mathrm{~h} \mathrm{~g}^{-1}$ and the $200^{\text {th }}$ cycle is $610 \mathrm{~mA} \mathrm{~h} \mathrm{~g}^{-1}$, which clearly indicate the good cyclability of the S-CNTs@rGO.

Electrochemical impedance spectroscopy (EIS) measurements were carried out to study the electrode's superior electrochemical performance and the kinetics properties. Fig. 7 shows the Nyquist plots of the fresh 3D S-CNTs@rGO and 3D S/rGO electrodes as well as the equivalent circuit model. The intersections of the semicircle at the $Z_{\text {real }}$-axis in the high frequency region, which was denoted as $R_{1}$, were attributed to the internal resistance, associated to the bulk resistance of electrolyte and electrode. The $R_{1}$ of the S-CNTs@rGO and S/rGO were 2.3 and $6.7 \Omega$ respectively, which indicated the improvement of electronic conductivity with embedded CNTs in the electrodes. It was found that $R_{2}$ corresponded to the charge transfer resistance. ${ }^{38}$ Simultaneously, the slope of the low frequency tail was approaching a typical Warburg behavior characteristic of semi-infinite diffusion. ${ }^{43}$ The semicircle diameter of the S-CNTs@rGO was smaller than that of S/rGO, which suggested an enhancement of the electron and ion transfer in the electrode/ electrolyte interface resulting from the embedded CNTs in the rGO layer. ${ }^{44}$ And the good conductivity of CNTs@rGO can be responsible for the excellent electrochemical performance.

\section{Conclusions}

A novel eco-friendly and non-toxic in situ method, using ethanol and element sulfur, has been applied in synthesizing the binder-free and free-standing 3D S-CNTs@rGO electrode. First, $S$ nano particles with diameter in the range of $20-200 \mathrm{~nm}$ were obtained by the method based on insolubility between polar materials and nonpolar materials. Second, through this method, nano $\mathrm{S}$ particles can be anchored or embedded in the CNTs@rGO layer without severe agglomeration problem, which minimize the loss of lithium polysulfides to the electrolyte. Moreover, the embedded CNTs in the basal plane of rGO can provide natural electron pathways that improve the conductivity of rGO. In addition, we expect that our strategy to synthesize 3D S-CNTs@rGO composites will arouse interest of designing cathode structure for $\mathrm{Li}-\mathrm{S}$ batteries with a more eco-friendly and non-toxic method.

\section{Conflicts of interest}

There are no conflicts to declare.

\section{Acknowledgements}

This work is supported by the Natural Science Foundation of China (No. 51373096), and Basic Research Field of Shanghai Science and Technology Innovation Program (No. 16JC1401500). Instrumental Analysis Center of Shanghai Jiao Tong University and National Engineering Research Center for Nanotechnology are gratefully acknowledged for assisting with relevant analyses.

\section{References}

1 Y. X. Yin, S. Xin, Y. G. Guo and L. J. Wan, Angew. Chem., Int. Ed. Engl., 2013, 52, 13186-13200.

2 H. Lu, W. Qin, J. Zhou, J. Jian, S. Lu, X. Wu, G. Fan, P. Gao and B. Liu, Nanoscale, 2017, 9, 5141-5147.

3 P. G. Bruce, S. A. Freunberger, L. J. Hardwick and J. M. Tarascon, Nat. Mater., 2011, 11, 19-29.

4 S.-E. Cheon, J.-H. Cho, K.-S. Ko, C.-W. Kwon, D.-R. Chang, H.-T. Kim and S.-W. Kim, J. Electrochem. Soc., 2002, 149, A1437.

5 B. Dunn, H. Kamath and J.-M. Tarascon, Science, 2011, 334, 928-935.

6 X. Ji, K. T. Lee and L. F. Nazar, Nat. Mater., 2009, 8, 500-506.

7 X. Ji, K. T. Lee and L. F. Nazar, Nat. Mater., 2009, 8, 500-506.

8 L. Ji, M. Rao, S. Aloni, L. Wang, E. J. Cairns and Y. Zhang, Energy Environ. Sci., 2011, 4, 5053-5059.

9 J. T. Lee, Y. Zhao, S. Thieme, H. Kim, M. Oschatz, L. Borchardt, A. Magasinski, W. I. Cho, S. Kaskel and G. Yushin, Adv. Mater., 2013, 25, 4573-4579.

10 L. Ji, M. Rao, H. Zheng, L. Zhang, Y. Li, W. Duan, J. Guo, E. J. Cairns and Y. Zhang, J. Am. Chem. Soc., 2011, 133, 18522-18525.

11 J. Guo, Y. Xu and C. Wang, Nano Lett., 2011, 11, 4288-4294. 12 X.-B. Cheng, J.-Q. Huang, Q. Zhang, H.-J. Peng, M.-Q. Zhao and F. Wei, Nano Energy, 2014, 4, 65-72.

13 G. Zhou, D.-W. Wang, F. Li, P.-X. Hou, L. Yin, C. Liu, G. Q. M. Lu, I. R. Gentle and H.-M. Cheng, Energy Environ. Sci., 2012, 5, 8901-8906.

14 D. Rao, Y. Wang, L. Zhang, S. Yao, X. Qian, X. Xi, K. Xiao, K. Deng, X. Shen and R. Lu, Carbon, 2016, 110, 207-214. 
15 G. Zhou, S. Pei, L. Li, D. W. Wang, S. Wang, K. Huang, L. C. Yin, F. Li and H. M. Cheng, Adv. Mater., 2014, 26, 625-631.

16 J.-Z. Wang, L. Lu, M. Choucair, J. A. Stride, X. Xu and H.-K. Liu, J. Power Sources, 2011, 196, 7030-7034.

17 R. Elazari, G. Salitra, A. Garsuch, A. Panchenko and D. Aurbach, Adv. Mater., 2011, 23, 5641-5644.

18 S. Lu, Y. Chen, X. Wu, Z. Wang and Y. Li, Sci. Rep., 2014, 4, 4629.

19 Y. Chen, S. Lu, J. Zhou, X. Wu, W. Qin, O. Ogoke and G. Wu, J. Mater. Chem. A, 2017, 5, 102-112.

20 Y. Chen, S. Lu, J. Zhou, W. Qin and X. Wu, Adv. Funct. Mater., 2017, 27, 1700987.

21 W. Ni, J. Cheng, X. Li, Q. Guan, G. Qu, Z. Wang and B. Wang, RSC Adv., 2016, 6, 9320-9327.

22 C. Wang, X. Wang, Y. Wang, J. Chen, H. Zhou and Y. Huang, Nano Energy, 2015, 11, 678-686.

23 Y.-L. Ding, P. Kopold, K. Hahn, P. A. van Aken, J. Maier and Y. Yu, Adv. Funct. Mater., 2016, 26, 1112-1119.

24 G. Hu, C. Xu, Z. Sun, S. Wang, H. M. Cheng, F. Li and W. Ren, Adv. Mater., 2016, 28, 1603-1609.

25 D. Yang, W. Ni, J. Cheng, Z. Wang, T. Wang, Q. Guan, Y. Zhang, H. Wu, X. Li and B. Wang, Appl. Surf. Sci., 2017, 413, 209-218.

26 Y.-E. Shin, Y. J. Sa, S. Park, J. Lee, K.-H. Shin, S. H. Joo and H. Ko, Nanoscale, 2014, 6, 9734-9741.

27 Y. Chen, S. Lu, X. Wu and J. Liu, J. Phys. Chem. C, 2015, 119, 10288-10294.

28 Z. Chen, H. Li, R. Tian, H. Duan, Y. Guo, Y. Chen, J. Zhou, C. Zhang, R. Dugnani and H. Liu, Sci. Rep., 2016, 6, 27365.

29 Q. Zeng, H. Li, H. Duan, Y. Guo, X. Liu, Y. Zhang and H. Liu, RSC Adv., 2015, 5, 13430-13436.
30 W. Ahn, M. H. Seo, Y. S. Jun, D. U. Lee, F. M. Hassan, X. Wang, A. Yu and Z. Chen, ACS Appl. Mater. Interfaces, 2016, 8, 1984-1991.

31 X. Ye, J. Ma, Y.-S. Hu, H. Wei and F. Ye, J. Mater. Chem. A, 2016, 4, 775-780.

32 C. Wang, K. Su, W. Wan, H. Guo, H. Zhou, J. Chen, X. Zhang and Y. Huang, J. Mater. Chem. A, 2014, 2, 5018.

33 R. Li, M. Zhang, Y. Li, J. Chen, B. Yao, M. Yu and G. Shi, Phys. Chem. Chem. Phys., 2016, 18, 11104-11110.

34 G. Li, J. Sun, W. Hou, S. Jiang, Y. Huang and J. Geng, Nat. Commun., 2016, 7, 10601.

35 J. Wang, S. Cheng, W. Li, S. Zhang, H. Li, Z. Zheng, F. Li, L. Shi, H. Lin and Y. Zhang, J. Power Sources, 2016, 321, 193-200.

36 J.-Q. Huang, Z.-L. Xu, S. Abouali, M. Akbari Garakani and J.-K. Kim, Carbon, 2016, 99, 624-632.

37 C. Wu, L. Fu, J. Maier and Y. Yu, J. Mater. Chem. A, 2015, 3, 9438-9445.

38 G. Zhou, E. Paek, G. S. Hwang and A. Manthiram, Nat. Commun., 2015, 6, 7760.

39 Z. Wang, Y. Dong, H. Li, Z. Zhao, H. B. Wu, C. Hao, S. Liu, J. Qiu and X. W. Lou, Nat. Commun., 2014, 5, 5002.

40 G. Zhou, L.-C. Yin, D.-W. Wang, L. Li, S. Pei, I. R. Gentle, F. Li and H.-M. Cheng, ACS Nano, 2013, 7, 5367-5375.

41 L. Zhang, L. Ji, P. A. Glans, Y. Zhang, J. Zhu and J. Guo, Phys. Chem. Chem. Phys., 2012, 14, 13670-13675.

42 L. Sun, M. Li, Y. Jiang, W. Kong, K. Jiang, J. Wang and S. Fan, Nano Lett., 2014, 14, 4044-4049.

43 J. He, Y. Chen, P. Li, F. Fu, Z. Wang and W. Zhang, J. Mater. Chem. A, 2015, 3, 18605-18610.

44 J. Yang, F. Chen, C. Li, T. Bai, B. Long and X. Zhou, J. Mater. Chem. A, 2016, 4, 14324-14333. 